\title{
How do we treat small cell anal cancer?
}

\begin{abstract}
Background: We report the rare case of a small cell cancer of the anal canal.

Case report: The patient presented with a locally advanced small cell anal cancer and was treated following the guidelines of locally advanced small cell cancer of the lung. While she achieved radiologic complete response for 9months, her local symptoms were predictive of local recurrence.

Conclusion: Small cell cancer of the anal canal is an extrapulmonary small-cell carcinoma. There are currently no level 1 evidence guidelines for treatment of these rare tumors. Further characterization of the molecular profile and treatment options is sorely needed.
\end{abstract}

Keywords: small cell, anal cancer, chemotherapy, radiotherapy
Volume 4 Issue I - 2017

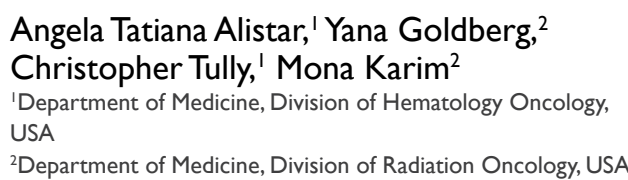

Correspondence: Angela Alistar, Atlantic Medical Group, Morristown Medical Center, 100 Madison Ave Carol G. Simon Cancer Center, Morristown, NJ 07962, USA, Tel 973.97I.7960, Email angela.alistar@atlantichealth.org

Received: June 28, 2017| Published: August 18, 2017
Abbreviations: EPSCC, extrapulmonary small-cell carcinoma; SCLC, small-cell lung carcinoma; LD, limited disease; ED, extensive disease; MRI, magnetic resonance imaging

\section{Background}

Extrapulmonary small-cell carcinoma (EPSCC) has been increasingly recognized as a clinicopathologic entity with a biological behavior and prognosis distinct from small-cell lung carcinoma (SCLC). EPSCC have an aggressive natural history that is characterized by early, widespread metastases, likely presenting as systemic disease at diagnosis. Even patients that present with a loco regional disease are likely to relapse and have a poor prognosis. ${ }^{1}$ In different studies, the median survival for limited and extensive disease ranges from 1.4 to $3.5 y$ years and 8 to 12 months, respectively. ${ }^{2,3}$ Extrapulmonary small cell carcinomas are uncommon, with an incidence of approximately $0.4 \%{ }^{4}$ The oesophagus is the most common primary site followed by colorectal. ${ }^{5}$ Less than $1 \%$ of anal cancers are small cell carcinomas-the most common being squamous cell carcinomas (74\%) and $19 \%$ are adenocarcinomas. ${ }^{6}$

\section{Clinical scenario}

We describe our experience and management of a patient with an EPSCC, localized small cell anal carcinoma. The patient is a 36yearold female with no significant past medical history, who presented with severalmonths of worsening rectal pain. Her family history includes three uncles with an unknown type of cancer. On exam under anesthesia, she was found to have an anterior ulcerating lesion, measuring $3 \times 4 \mathrm{~cm}$, extending proximally from the dentate line. Pathology from a biopsy revealed a poorly differentiated neuroendocrine carcinoma, consistent with small cell carcinoma. The tumor had lymphovascular invasion and a high Ki67, and was positive for CK7, synaptophysin, CD56, chromogranin, P16 and TTF1. Flow cytometry was negative. She had a PET/CT which revealed hypermetabolic thickening of the anorectal area with an SUV of 15.9, as well as enlargement and hypermetabolic activity in multiple pelvic lymph nodes, including left perirectal, left internal iliac, left external iliac and common iliac areas (Figure 1).

She did not undergo imaging of her brain as she didn't have neurologic symptoms. She was then started on concurrent Cisplatin/
Etoposide chemotherapy and pelvic radiation. She received whole pelvic radiation to $45 \mathrm{~Gy}$, including the primary tumor, inguinal and bilateral pelvic nodes up through the lower para-aortic chain, followed by a conedown to 54 Gray to the PET-avid left pelvic nodes and the primary tumor. This was followed by a final conedown to deliver 5.4 additional Gray to the primary tumor, for a total dose 59.4 Gray to the anorectal tumor. During treatment, she had significant skin toxicity in the gluteal/labial area and inguinal folds with moist desquamation and pain treated with supportive care. She was able to maintain her weight during the radiation therapy, and completed with only a oneday treatment break in the final week for skin toxicity.
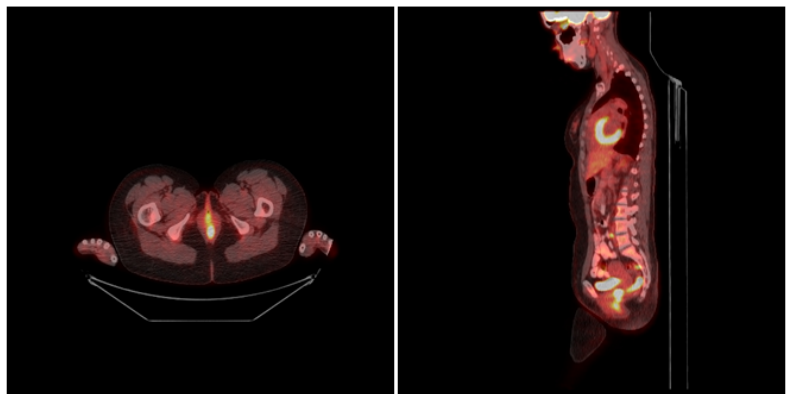

Figure I Initial PET/CT showing SUV uptake in anorectal tumor and left iliac nodes.

The patient went on to complete a total of six cycles of Cisplatin/ Etoposide. She developed mild neuropathy, occasional loose stools and depression (treated with Xanax and Effexor). Her rectal pain decreased but did not resolve after radiation, and she continued on narcotics. A restaging PET scan 2months after the completion of chemotherapy and $3 \frac{1}{2}$ months after the completion of RT showed complete resolution of hypermetabolism (Figure 2).

She continued to experience rectal and lower pelvic pain, and had a $\mathrm{CT}$ chest/abdomen/pelvis two months after the PET scan which again showed no evidence of disease. The pelvic and rectal pain persisted. Four months after the negative restaging $\mathrm{CT}$ and 9months after the completion of all therapy, she had a pelvic MRI which showed a recurrent anterior rectal wall mass in the area of the original primary tumor and multiple enlarged right pelvic lymph nodes. A PET scan a week later confirmed hypermetabolism in the distal rectum and the 
left (not right) external iliac, internal iliac and common iliac areasthese areas had all been within the radiation boost region (Figure 3). She then resumed chemotherapy with carboplatin/etoposide. Two cycles are planned before further restaging scans.
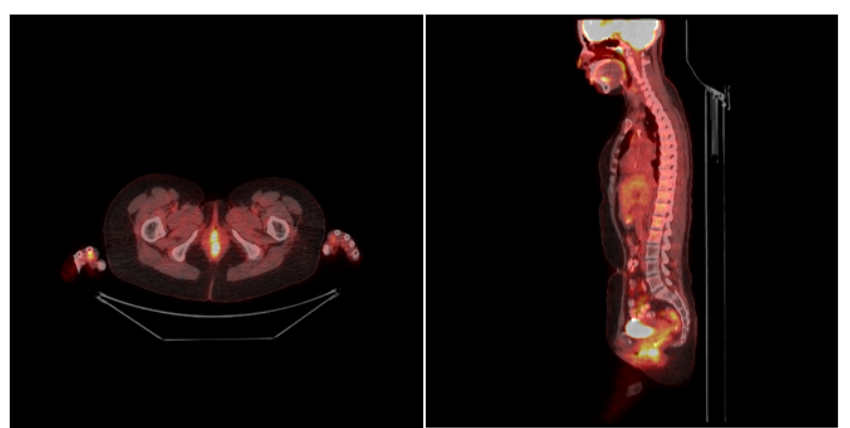

Figure 2 PET/CT 2 months after completion of chemoradiation showing complete resolution of uptake.
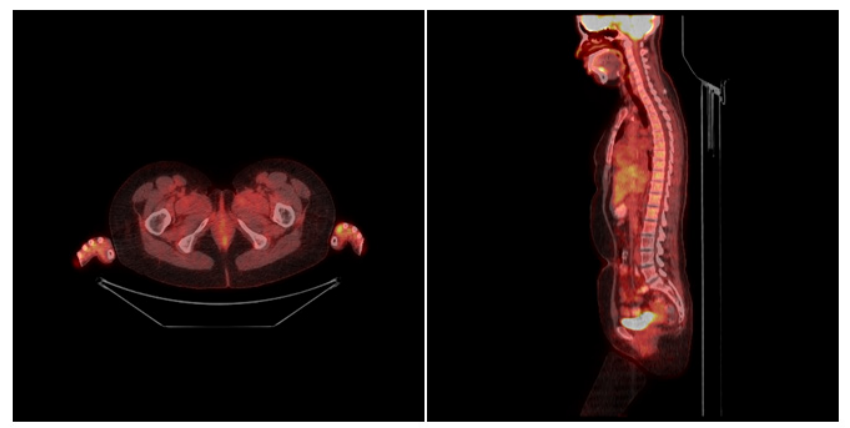

Figure $3 \mathrm{PET} / \mathrm{CT}$ nine months after completion of therapy with recurrent hypermetabolism in anorectal area and left iliac nodes.

\section{Discussion}

Currently, no specific staging system for EPSCC has been established. The most frequently used staging system is Veterans' Administration Lung Study Group, consisting of limited disease (LD), defined as tumor contained within a localized anatomic region, with or without involved regional lymphadenopathy; and extensive disease (ED), defined as tumor outside the loco-regional boundaries). ${ }^{5}$ The first step in management is confirmation of the histological diagnosis as well as the exclusion of a primary lung tumor. Magnetic resonance imaging (MRI) of the brain is recommended if neurologic symptoms are present. Bone marrow biopsy is indicated only if there are abnormal blood counts or findings on the peripheral smear, without other evidence of disseminated disease. Based on the results of such evaluation, patients with disease confined to a primary site and/ or regional nodes are classified as having limited disease, while all others are considered to have extensive disease. The current clinical management of the EPSCC follows the guidelines of the SCLC.

A study published in 2010 by Brennan et al., ${ }^{3}$ aimed to determine whether extra pulmonary small cell carcinomas should be managed using protocols similar to those for small cell lung cancers. Based on the retrospective single institution analysis of 120 eligible patients ( $70 \%$ with limited disease and $30 \%$ with extensive disease) they concluded that patients with extrapulmonary small-cell carcinoma experience short survival with early distant metastasis. The median survival for aggressively treated EPSCC patients with LD was similar to the median survival of 1.2 years for a cohort of 90 patients with
SCLC treated with definitive chemoradiation at the same institution over a comparable time period. The treatment modalities offered to patients were chemotherapy, radiotherapy and surgery. The study confirms that the rate of brain metastasis was lower than for patients with small cell lung carcinoma. ${ }^{2}$ This is consistent with other reports in the literature. ${ }^{4,7,8}$ While this finding doesn't prompt PCI (prophylactic cranial irradiation) for all the patients with EPSCC, it poses the question whether initial staging for locally advanced EPSCC should include MRI of the brain in the absence of neurological symptoms. An exception would be EPSCC originating from the head and neck region which is associated with a higher incidence of brain metastasis, justifying addition of PCI (prophylactic cranial irradiation).

\section{Conclusion}

In the absence of prospective clinical trials, retrospective studies represent the evidence we use to make clinical decisions in practice. While cure is possible for a small number of patients with EPSCC with limited disease it is hard to determine if the rare, almost anecdotal better outcomes are due to a more favorable biology versus aggressive treatment. Complete staging is important to inform the prognosis and treatment of the patients. In the era of precision oncology understanding the molecular biology and mutational profile of patients with this disease will open opportunities for targeted therapies and clinical trials. Molecular characterization of EPSCC will be the best strategy to inform potential therapeutic strategy in this rare disease.

\section{Acknowledgements}

None.

\section{Conflict of interest}

Author declares that there is no conflict of interest.

\section{References}

1. Beahrs OH, Wilson SM. Carcinoma of the anus. Ann Surg. 1976;184(4):422-428.

2. Brennan SM, Gregory DL, Stillie A, et al. Should extrapulmonary small cell cancer be managed like small cell lung cancer? Cancer 2010;116(4):888-895.

3. Brenner B, Tang LH, Klimstra DS, et al. Small-cell carcinomas of the gastrointestinal tract: a review. J Clin Oncol. 2004;22(13):2730-2739.

4. Cicin I, Karagol H, Uzunoglu S, et al. Extrapulmonary small-cell carcinoma compared with small-cell lung carcinoma: a retrospective single-center study. Cancer. 2007;110(5):1068-1076.

5. Ochsenreither S, Marnitz-Schultze S, Schneider A, et al. Extrapulmonary small cell carcinoma (EPSCC): 10years' multi-disciplinary experience at Charite. Anticancer Res. 2009;29(8):3411-3415.

6. Soto DE, Eisbruch A. Limited-stage extrapulmonary small cell carcinoma: outcomes after modern chemotherapy and radiotherapy. Cancer $J$. 2007;13(4):243-246.

7. Viswanathan AN, Deavers MT, Jhingran A, et al. Small cell neuroendocrine carcinoma of the cervix: outcome and patterns of recurrence. Gynecol Oncol. 2004;93(1):27-33.

8. Walenkamp AM, Sonke GS, Sleijfer DT. Clinical and therapeutic aspects of extrapulmonary small cell carcinoma. Cancer Treat Rev. 2009;35(3):228-236. 